This item was submitted to Loughborough's Research Repository by the author.

Items in Figshare are protected by copyright, with all rights reserved, unless otherwise indicated.

\title{
Biopolymer dynamics driven by helical flagella
}

PLEASE CITE THE PUBLISHED VERSION

https://doi.org/10.1103/PhysRevFluids.2.113102

PUBLISHER

(C) American Physical Society

VERSION

VoR (Version of Record)

\section{PUBLISHER STATEMENT}

This work is made available according to the conditions of the Creative Commons Attribution-NonCommercialNoDerivatives 4.0 International (CC BY-NC-ND 4.0) licence. Full details of this licence are available at: https://creativecommons.org/licenses/by-nc-nd/4.0/

\section{LICENCE}

CC BY-NC-ND 4.0

\section{REPOSITORY RECORD}

Balin, Andrew K., Andreas Zottl, Julia M. Yeomans, and Tyler Shendruk. 2019. "Biopolymer Dynamics Driven by Helical Flagella". figshare. https://hdl.handle.net/2134/36371. 


\title{
Biopolymer dynamics driven by helical flagella
}

\author{
Andrew K. Balin, ${ }^{1}$ Andreas Zöttl, ${ }^{1}$ Julia M. Yeomans, ${ }^{1}$ and Tyler N. Shendruk ${ }^{1,2, *}$ \\ ${ }^{1}$ Rudolf Peierls Centre for Theoretical Physics, 1 Keble Road, University of Oxford, \\ Oxford OX1 3NP, United Kingdom \\ ${ }^{2}$ Rockefeller University, 1230 York Avenue, New York, New York 10021, USA
}

(Received 25 May 2017; published 16 November 2017)

\begin{abstract}
Microbial flagellates typically inhabit complex suspensions of polymeric material which can impact the swimming speed of motile microbes, filter feeding of sessile cells, and the generation of biofilms. There is currently a need to better understand how the fundamental dynamics of polymers near active cells or flagella impacts these various phenomena, in particular, the hydrodynamic and steric influence of a rotating helical filament on suspended polymers. Our Stokesian dynamics simulations show that as a stationary rotating helix pumps fluid along its long axis, polymers migrate radially inward while being elongated. We observe that the actuation of the helix tends to increase the probability of finding polymeric material within its pervaded volume. This accumulation of polymers within the vicinity of the helix is stronger for longer polymers. We further analyze the stochastic work performed by the helix on the polymers and show that this quantity is positive on average and increases with polymer contour length.
\end{abstract}

DOI: 10.1103/PhysRevFluids.2.113102

\section{INTRODUCTION}

While the physics of swimming microbes in Newtonian viscous fluids has been well characterized, attention has recently turned toward understanding how active microorganisms behave in more biologically relevant media where the presence of large biopolymers, elastic filaments, or exopolymer secretions can dictate dynamics. In such complex fluids, motility enhancement [1-6] and retardation $[3,4,7-11]$ have both been reported for various biological swimmers. Theoretical studies exploring swimming in continuous viscoelastic media yield model- or parameter-dependent results [3-5]. Invariably, these studies concentrate on continuum models of viscoelasticity, and as such cannot provide a full insight into the specific microscopic mechanisms of interaction between single polymers and the flagella of the swimmers.

Swimming dynamics may indeed be affected by such interactions due in part to the comparatively similar length and time scales of cells and biopolymeric material in vivo. One hypothesis that has been applied to the swimming of $E$. coli in dilute polymer solutions is that a time-scale separation between the fast rotation of the bacterial flagellum and slow relaxation of the polymers effectively depletes the flagellum's local environment of polymeric material as it clears its surrounding volume [12]. Hence, the flagellum experiences an effective viscosity that can be markedly different from that perceived by the more slowly counter-rotating cell body. However, the microscopic assumptions underlying this hypothesis require more concrete justification. Another hypothesis posits that the curved streamlines that wind around the helical flagellum produce shear flows that stretch individual polymers, resulting in elastic stresses which stabilize — hence speeding up — the cell's swimming [6]. It is recognized that this hypothesis relies on the expectation that the swimmer generates sufficient strain rates to stretch polymer molecules and raises the question of how large biopolymers interact with actively actuated filaments such as flagella on the single-polymer level.

Additionally, there are separate phenomena in which the interactions between large polymers and active microorganisms are important but have yet to be studied on this microscopic level. For

*tshendruk@ rockefeller.edu 


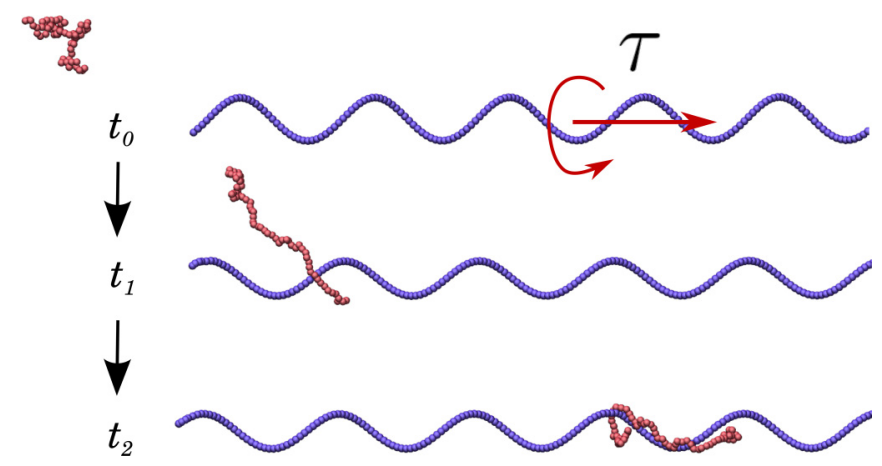

FIG. 1. Snapshots from a single simulation of a rotating helix and an advected polymer taken at times $t_{0}<t_{1}<t_{2}$. A torque $\boldsymbol{\tau}$ is distributed uniformly on the helix and, as it rotates, it generates a pumping flow. The polymer is drawn in radially and axially toward the helix, stretching in the process due to the induced shear flow. Once captured, it winds around the helix and is pumped axially in the direction of the average flow.

instance, various microbes have long been known to enhance filter feeding by employing their flagella or cilia to generate feeding currents that carry detritus and nutrients toward the cell body [13]. While motile neutrally buoyant planktonic bacteria [14] swim force free [15,16], these sessile microbes tether themselves in order to exert non-negligible net forces on the surrounding medium $[17,18]$. Aggregations of such cells can collectively produce millimeter-scale fluid flows to actively combat variations in the nutrient concentrations [19]. While hydrodynamic attraction of small nutrients has been considered [20], the interaction of macromolecular polymers with sessile flagellates has yet to be explored in detail. A question that remains open is whether the filter feeding of large polymers progresses similarly to small tracer particles, or-as with swimming-whether the dynamics of individual polymers enhances or reduces a tethered cell's ability to draw in polymeric material toward it.

In this work, we explore how macromolecular biopolymers hydrodynamically interact with an active helical pump that transports fluid by external actuation using coarse-grained molecular dynamics simulations. A snapshot from our simulations is shown in Fig. 1. We first construct a simple Stokesian dynamics model of a rotating helix in a bulk fluid and justify the further use of this simplified model by comparing the flow field in the vicinity of the helix to that of a more specific biological model system comprised of a wall-tethered bacterium in a coarse-grained fluid. We show that the Stokesian dynamics scheme captures the relevant near-field physics of flow around a helix and proceed to study the dynamics of single polymers in its vicinity. Our results show that model polymers are hydrodynamically drawn radially inward and are elongated by the high shear rate. The polymers are then transported along the direction of the fluid flow while remaining elongated and wrap around the helix before being deposited downstream.

We also study the stochastic energetics of the helix to show that the helix actively performs work on the polymer, driving it to the higher free-energy state of elongation which we observe. This work is positive on average, suggesting that the energy transferred to the polymer through hydrodynamic interactions is dissipated and not elastically transferred back to the helix.

\section{METHODS}

\section{A. Stokesian dynamics simulation}

We employ a simulation scheme incorporating hydrodynamic interactions [21], referred to as Stokesian dynamics (SD), to study the behavior of a polymer in response to a steadily rotating helical filament. Both the polymer chain and the rotating helix are composed of sets of spherical 
particles whose positions $\left\{\boldsymbol{r}_{i}\right\}$ are updated according to Langevin dynamics due to forces $\left\{\boldsymbol{f}_{i}\right\}$ and thermal fluctuations $\left\{\boldsymbol{\xi}_{i}\right\}$ :

$$
\begin{gathered}
\dot{\boldsymbol{r}}_{i}=\sum_{j}\left(\mathrm{k}_{B} T \frac{\partial \boldsymbol{\mu}_{i j}}{\partial \boldsymbol{r}_{j}}+\boldsymbol{\mu}_{i j} \boldsymbol{f}_{j}\right)+\boldsymbol{\xi}_{i}(t), \\
\left\langle\boldsymbol{\xi}_{i}(t) \boldsymbol{\xi}_{j}\left(t^{\prime}\right)\right\rangle=2 \mathrm{k}_{B} T \boldsymbol{\mu}_{i j} \delta\left(t-t^{\prime}\right) .
\end{gathered}
$$

Equations (1) and (2) are coupled in two ways: (i) The total force $\boldsymbol{f}_{i}\left(\left\{\boldsymbol{r}_{j}\right\}\right)$ acting on particle $i$ will in general contain a contribution due to pairwise interactions with nearby particles and (ii) hydrodynamic coupling between particles $i$ and $j$ is captured by the mobility tensor $\boldsymbol{\mu}_{i j}\left(\boldsymbol{r}_{i}, \boldsymbol{r}_{j}\right)$, which accounts for the advection of particle $i$ due to the flow field created by forces $\boldsymbol{f}_{j}$ acting on particle $j$. The fluid medium is responsible for dissipating the momentum of the particles, demanding the fluctuating forces obey the fluctuation-dissipation relation [Eq. (2)], which correlates the fluctuations experienced by two widely separated particles.

We use the Rotne-Prager-Yamakawa (RPY) tensor for the mobility [22,23],

$$
\frac{\boldsymbol{\mu}_{i j}}{\mu_{0}}= \begin{cases}\frac{3 a}{4 r_{i j}}\left(\boldsymbol{I}+\hat{\boldsymbol{r}}_{i j} \hat{\boldsymbol{r}}_{i j}\right)+\frac{a^{3}}{2 r_{i j}^{3}}\left(\boldsymbol{I}-3 \hat{\boldsymbol{r}}_{i j} \hat{\boldsymbol{r}}_{i j}\right) & \text { for } r_{i j} \geqslant 2 a, \\ \left(1-\frac{9 r_{i j}}{32 a}\right) \boldsymbol{I}+\frac{3}{32} \frac{r_{i j} a}{a} \hat{\boldsymbol{r}}_{i j} \hat{\boldsymbol{r}}_{i j} & \text { otherwise, }\end{cases}
$$

for $i \neq j$ and where $\boldsymbol{r}_{i j}=\boldsymbol{r}_{j}-\boldsymbol{r}_{i}, \mu_{0}=1 / 6 \pi \eta a$ is the Stokes mobility of a sphere with radius $a$ immersed in a fluid with viscosity $\eta$ and $\boldsymbol{I}$ is the identity matrix. The self-mobility of particle $i$ is simply $\boldsymbol{\mu}_{i i}=\mu_{0} \boldsymbol{I}$. The RPY tensor has the property that $\partial \boldsymbol{\mu}_{i j} / \partial \boldsymbol{r}_{j}=0$, which simplifies the force balance equation (1).

\section{B. Polymer model}

All pairs of particles experience a mutual repulsion that acts over a characteristic length scale $\sigma$ and is given by the Weeks-Chandler-Andersen (WCA) potential,

$$
\mathcal{H}_{\text {repel }}\left(r_{i j}\right)= \begin{cases}4 \epsilon\left[\left(\frac{\sigma}{r_{i j}}\right)^{12}-\left(\frac{\sigma}{r_{i j}}\right)^{6}\right]+\epsilon, & \text { if } r_{i j}<2^{1 / 6} \sigma, \\ 0, & \text { otherwise }\end{cases}
$$

The polymer is modeled as a chain of spherical beads, exerting pairwise attractive forces representing bonds between adjacent monomers. These bonding forces are calculated using the finitely extensible nonlinear elastic (FENE) potential [24],

$$
\mathcal{H}_{\text {bond }}\left(r_{i j}\right)=-\frac{1}{2} k_{\text {bond }} r_{0}^{2} \ln \left[1-\left(\frac{r_{i j}}{r_{0}}\right)^{2}\right],
$$

with Kremer-Grest parameters [25] $k_{\text {bond }}=30 \epsilon / \sigma^{2}$ and $r_{0}=1.5 \sigma$. We choose $\epsilon=\mathrm{k}_{B} T$ for the characteristic strength of the potentials, $\sigma$ for the spatial unit, and $\sigma^{2} / \mu_{0} \mathrm{k}_{B} T$ for the temporal unit. This allows us to set $\mathrm{k}_{B} T=1, \sigma=1, \mu_{0}=1$ hereafter. In these units, the bead diameter is $2^{1 / 6}$; hence $a=2^{-5 / 6}$, and the simulation time step is $\delta t=10^{-5}$. The conservative Hamiltonians $H_{\text {repel }}$ and $H_{\text {bond }}$ give rise to pairwise forces, $\boldsymbol{f}_{i}=-\boldsymbol{f}_{j}=-\nabla\left[\mathcal{H}_{\text {repel }}\left(r_{i j}\right)+\mathcal{H}_{\text {bond }}\left(r_{i j}\right)\right]$, which are the equal and opposite forces acting on particles $i$ and $j$.

For a three-dimensional system of $N$ particles, Eqs. (1) can be rewritten in nondimensional form as a $3 N \times 3 N$ matrix-vector difference equation involving time step $\delta t$,

$$
\delta \boldsymbol{r}=\boldsymbol{\mu} \boldsymbol{f} \delta t+\boldsymbol{b} \delta \boldsymbol{w},
$$

where $\delta \boldsymbol{w}$ is a random Gaussian vector with the properties $\langle\delta \boldsymbol{w}\rangle=\mathbf{0}$ and $\langle\delta \boldsymbol{w} \delta \boldsymbol{w}\rangle=2 \delta t \boldsymbol{I}$, and $\boldsymbol{b}$ is any matrix which satisfies $\boldsymbol{b} \boldsymbol{b}^{T}=\boldsymbol{\mu}$. We find $\boldsymbol{b}$ by computing the Cholesky decomposition of $\boldsymbol{\mu}$ and note that Cholesky decomposition requires $\boldsymbol{\mu}$ to be positive definite, which is ensured by the RPY 
tensor. Because the mobilities $\boldsymbol{\mu}_{i j}$ vary slowly with respect to the fastest time scales of the bond potentials, we update $\boldsymbol{\mu}$ once every 100 time steps.

\section{Rotating helix model}

The rotating helix comprises a set of particles whose individual positions are externally controlled by time-dependent forces that prescribe the shape, rotational frequency $\omega$, and translational speed $v$ of the helix. This is done by applying a rectifying force that opposes displacements of a constituent particle from its prescribed location via a stronger harmonic potential,

$$
\begin{gathered}
\mathcal{H}_{h}\left(\boldsymbol{r}_{i}, t\right)=\frac{1}{2} k_{h}\left[\boldsymbol{r}_{i}-\boldsymbol{r}_{i}^{0}(t)\right]^{2}, \\
\boldsymbol{r}_{i}^{0}=\left(\begin{array}{c}
R_{0} \cos \left(\kappa z_{i}^{0}-\omega t\right) \\
R_{0} \sin \left(\kappa z_{i}^{0}-\omega t\right) \\
z_{i}^{0}+v t
\end{array}\right) .
\end{gathered}
$$

The prescribed positions $\left\{\boldsymbol{r}_{i}^{0}\right\}$ trace a helix along $\hat{z}$, with an imposed radius $R_{0}$, and pitch $\kappa$ [26]. For the helix potential strength, we use $k_{h}=70 \epsilon$. By applying the constraint that the target positions must be separated by diameter $2 a$ in space, the required spacing in $z$ is approximated by $z_{i}^{0}=2 a / \sqrt{1+\kappa^{2} R^{2}}$. We impose a constant angular rotation rate $\omega$ about the $\hat{z}$ axis, and enforce stationarity by setting $v=0$ along the $\hat{z}$ axis. This model is adequate for reproducing the hydrodynamics of a rigid helix. Alternatively, modeling the helix as a semiflexible polymer would require solving bonding angle and dihedral angle potentials with large stiffnesses [27], in turn requiring $\delta t$ to be many orders of magnitude below the shortest time scale of interest, which for our purposes is the relaxation time $\tau_{0} \sim a^{2} / \mu_{0} \mathrm{k}_{B} T$ of the SD beads.

The helix particles (labeled by subscript $i$ ) are initialized in their target positions at $t=0$, and then the helix as a whole relaxes into a steady state after a short transient period. The steady state differs slightly from the target shape due to (i) a viscous, drag-induced phase lag behind their target position, causing a shrinkage in their radial coordinate, which can shown to be $R=R_{0} / \sqrt{1+\left(\mu_{0} \omega / k_{h}\right)^{2}}$ when no hydrodynamic interactions are present, and (ii) an additional collective displacement along $z$ due to chiral asymmetry in the hydrodynamic interactions $\sum_{j(\neq i)} \boldsymbol{\mu}_{i j} \cdot \boldsymbol{f}_{j}$ with the other helix particles. For $v=0$, these displacements $\delta z_{i}$ are counteracted by a net force on the helix $-\frac{\partial \mathcal{H}_{h}}{\partial z}=-N_{h} k_{h} \delta z$, which is imparted to the fluid in the $\hat{z}$ direction.

With this control over $\omega$ and $v$, we can drag, rotate, or apply some combination of translation and rotation to the helix. The imposed rotation and translation implies an external axial force and torque via the linear mobility relation

$$
\left(\begin{array}{c}
v \\
\omega
\end{array}\right)=\left(\begin{array}{ll}
\mu_{t t} & \mu_{t r} \\
\mu_{r t} & \mu_{r r}
\end{array}\right)\left(\begin{array}{l}
f_{z} \\
\tau_{z}
\end{array}\right)
$$

where the components $\mu_{t t}, \mu_{t r}=\mu_{r t}, \mu_{r r}$ are mobility coefficients for the helix as a whole and not to be confused with the Rotne-Prager tensor $\mu$. In general, the full mobility relation also includes off-axis force and torque components $f_{x, y}$ and $\tau_{x, y}$; however, these are small compared to $f_{z}$ and $\tau_{z}$, and because the potential $\mathcal{H}_{h}$ keeps the helix centered and aligned along $\hat{z}$, this results in small off-axis contributions to the fluid flow.

\section{HELIX HYDRODYNAMICS}

\section{A. Flow field generated by rotating helix in SD simulations}

We focus solely on a stationary helix $(v=0)$ rotating at some angular speed $\omega$. This implies a nonzero force $f_{z}=-\frac{\mu_{t r}}{\mu_{t t}} \tau_{z}$ must be imparted to the fluid, and the rotating helix acts as a pump. This model evokes the microscopic experiments on tethered bacteria [28,29] as well as many scaled-up experiments of tethered flagella-like filaments [30-34]. 


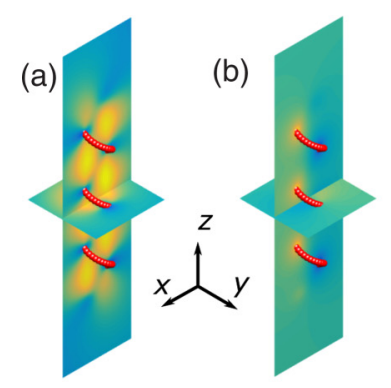

(e)

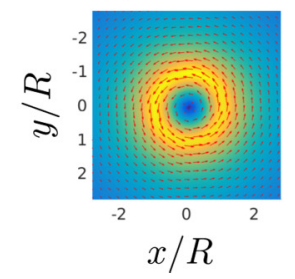

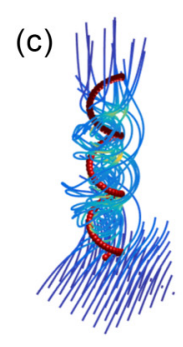

(f)
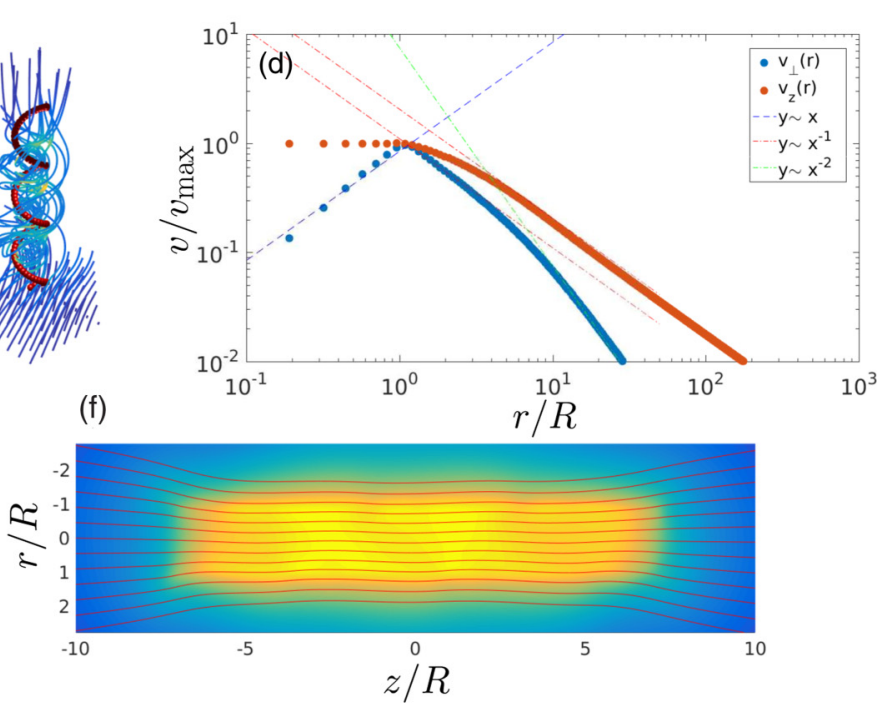

FIG. 2. [(a), (b)] Cross sections of the instantaneous flow field generated by a rotating helix, decomposed into components $v_{z}(\boldsymbol{r}, t)$ and $v_{\perp}(\boldsymbol{r}, t)$ respectively. (c) Streamlines of the full flow field $\boldsymbol{v}(\boldsymbol{r}, t)$ show both the net flow along $\hat{z}$ and the chiral winding caused by the rotation of the helix. (d) timeaveraged fields as a function of radial distance from helix center line, $\bar{v}_{z}(r, t)$ and $\bar{v}_{\perp}(r, t)$. Within the volume of the helical filament $(r<R)$, the fluid rotates like a rigid body, $v_{\perp} \propto r$, and flows axially at a constant rate $v_{r}=$ const. (e) Slice in the $x y$ plane of the mean rotational flow. (f) Slice in the $x z$ plane of the mean axial flow.

In the SD simulations of a helix on its own, we can evaluate the instantaneous flow field at any point $\boldsymbol{r}$ in space by summing the contributions that each particle in the simulation makes: $\boldsymbol{v}(\boldsymbol{r})=\sum_{i} \boldsymbol{\mu}\left(\boldsymbol{r}-\boldsymbol{r}_{i}\right) \cdot \boldsymbol{f}_{i}$, where $\boldsymbol{\mu}\left(\boldsymbol{r}^{\prime}\right)$ is given by Eq. (3) with $\boldsymbol{r}_{i j}=\boldsymbol{r}^{\prime}$. Figures 2(a)-2(c) offer a visualization of the instantaneous flow field surrounding a rotating helix. In Fig. 2(a), the axial component $v_{z}$ shows that the fluid is most strongly pumped in the interstitial volume of the helix, similar to the instantaneous axial flow field measured in experiments on a tethered rotating helix [34]. As the axial velocities of the helix beads are zero, $v_{z}$ must vanish at the helix surface. However, the transverse flow field $v_{\perp}$ is strongest at the helix surface as it must match the transverse velocity of the beads [Fig. 2(b)]. An instantaneous snapshot of the streamlines originating from a square grid in the $x y$ plane beneath the helix gives a visual sense of the chiral nature of the flow field [Fig. 2(c)].

By taking a time average $\overline{\boldsymbol{v}}(x, y, z)$ over a complete rotation of the helix, we can understand how the flow field varies in space in more detail. Figure 2(d) shows how $\bar{v}_{z}$ and $\bar{v}_{\perp}$ decay as a function of radial distance $r$ from the $z$ axis along which the helix lies. In the far field, we observe $\bar{v}_{z} \sim 1 / r$, which is the characteristic scaling expected from a point-force (stokeslet) response of an unbounded fluid. This is as expected, since we must apply a force $f_{z}$ on the helix such that $\mu_{t t} f_{z}+\mu_{t r} \tau_{z}=0$ by Eq. (9) to ensure the rotating helix remains stationary $(v=0)$. Hence, far away from the helix, the fluid responds as if subject to a point force.

The far-field scaling of the transverse velocity is the characteristic scaling for a rotlet, $\bar{v}_{\perp} \sim 1 / r^{2}$, which we expect to dominate the far-field flow created by an external torque rotating a body immersed in the fluid. However, in the intermediate region $(R<r<10 R)$, the transverse forces on the beads on the near side of the helix dominate over the oppositely directed forces on the far side, and therefore a stokeslet-like scaling $\bar{v}_{\perp} \sim 1 / r$ is seen.

Within the interior of the helix, we see the interesting scaling properties $\bar{v}_{z} \sim$ const, and $\bar{v}_{\perp} \sim r$, which show that on average the fluid inside the helix rotates about $\hat{z}$ and translates along $\hat{z}$ as a rigid body - though the instantaneous dynamics are more complicated. Figures 2(e)-2(f) show the 

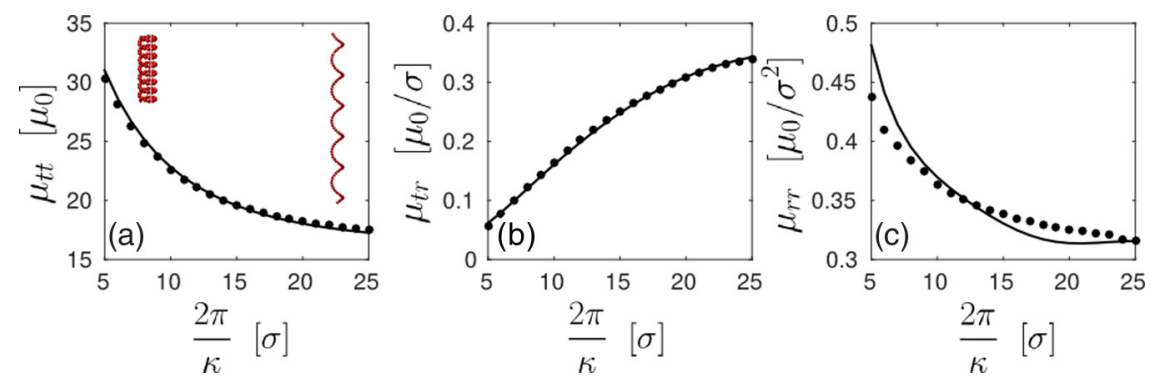

FIG. 3. The three components of the helix friction tensor as a function of pitch length $2 \pi / \kappa$. The data (black dots) are determined by measuring $f_{z}$, and $\tau_{z}$ separately as functions of $v$ and $\omega$ for different $\kappa$ in order to determine the matrix components $\mu_{t t}, \mu_{t r}$, and $\mu_{r r}$. The solid lines are calculated using analytical results from slender body theory (Eqs. (44) from Ref. [16]). Increasing the pitch length decreases the isotropic components of the friction tensor, while increasing the coupling between rotation and translation. Inset: The helix shapes for the extremal choices of pitch.

time-averaged flow fields. The transverse flow field is strongest in the annular region occupied by the helical filament itself, while the axial flow field is uniformly strong across the whole volume.

\section{B. Friction tensor of helical filament in SD simulations}

In order to measure the helix mobility, we conducted SD simulations of a helix (with no polymer present) while linearly ramping up either the velocity or angular velocity and keeping the other zero. In the first instance $\left(v=v_{0} t / \mathcal{T}, \omega=0\right)$ was imposed and in the second instance $\left(v=0, \omega=\omega_{0} t / \mathcal{T}\right)$ was imposed, where $v_{0}$ and $\omega_{0}$ are the target final velocities and the length of the simulation, $\mathcal{T}$, was sufficiently long to ensure that the system remained in a quasisteady state. In both cases, we measured $\left(f_{z}, \tau_{z}\right)$ in order to solve the linear system Eq. (9). We conducted this for a range of $\kappa \in\left(\frac{2 \pi}{25}, \frac{2 \pi}{5}\right)$ to measure how the friction components changed as a function of helix shape. The functional dependence of these coefficients on $\kappa$ can be derived analytically using slender-body theory $[35,36]$. The SD simulation results are in good agreement with theoretical predictions [16] as shown in Fig. 3.

We observe that as the pitch length increases, $\mu_{t t}$ and $\mu_{r r}$ decrease, while $\mu_{t r}$ increases. This tells us that the coupling between axial force and rotation (or conversely between applied torque and resulting translational speed) increases as the pitch length is increased over the range shown. This behavior can be understood intuitively by considering the limiting case of small pitch length $2 \pi / \kappa \rightarrow \sigma$, in which the filament resembles a cylinder which by symmetry must have a totally decoupled mobility relation.

The data for $\mu_{t t}$ and $\mu_{t r}$ fit very well to the slender-body prediction. However, while $\mu_{r r}$ qualitatively displays a similar dependence on pitch length to the analytic prediction, it appears to systematically deviate from the theory. As previously discussed, the steady-state shape of the helix realized in a simulation deviates slightly from its target shape [defined by Eq. (7)]. This effect of this is generally small, but it appears that $\mu_{r r}$ is the more sensitive to this dynamic remodeling than the other components of the mobility matrix. However, in the remainder of this study, we will only conduct simulations in which the helix parameters remain constant and so this discrepancy in $\mu_{r r}$ as a function of $\kappa$ does not affect our findings.

\section{Scope of rotating helix model}

Because our model helix remains in a fixed location in $\hat{z}$, it is neither force-free nor torque-free; hence its flow field will differ to that generated by a swimming cell in the far field. Artificial swimming magnetic ribbons [37,38] are arguably the most similar experimental realization of our system due to the net torque they impart, but unless they are stalled by an external force, they 

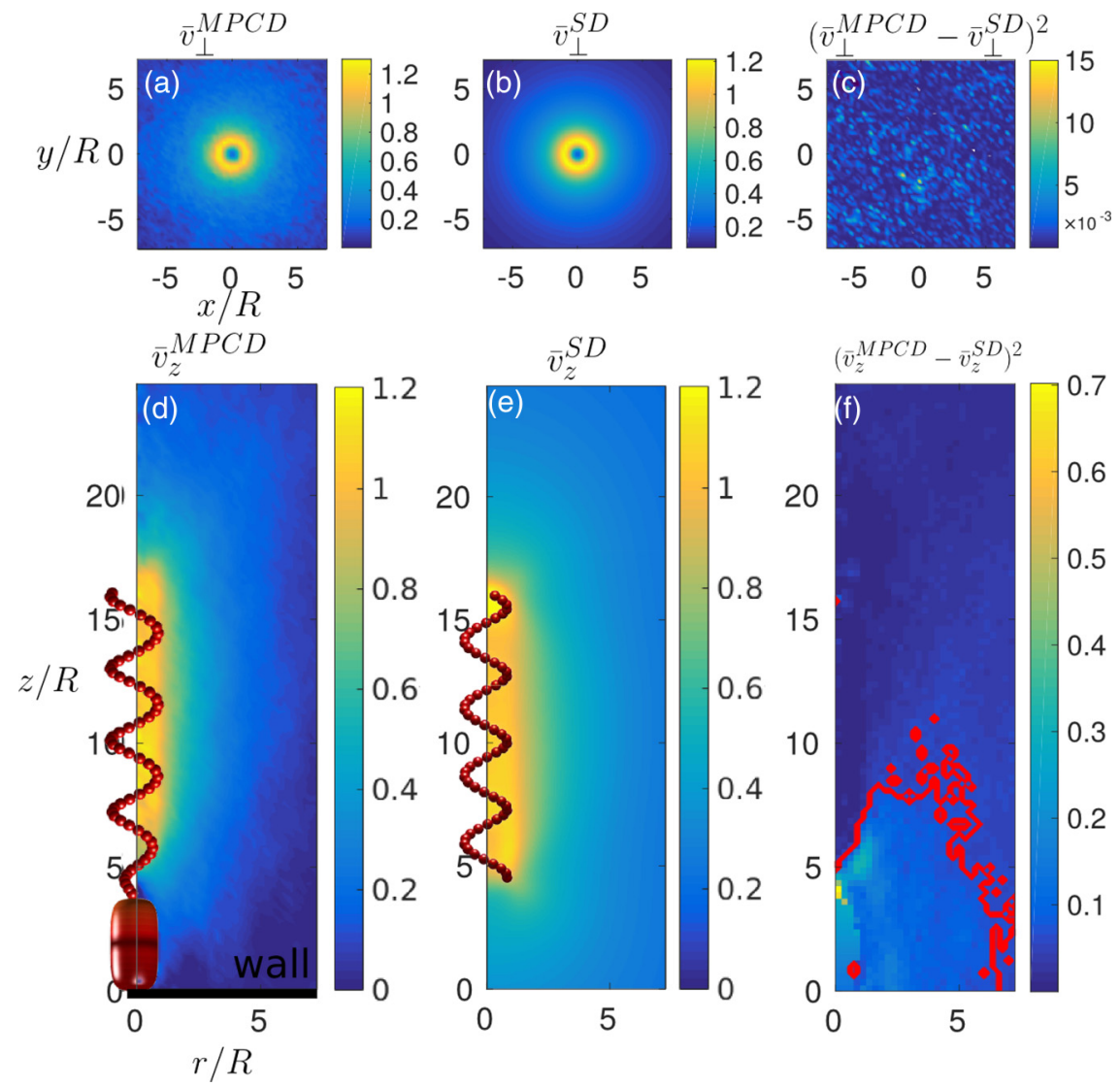

FIG. 4. [(a)-(c)] Comparison of the time-averaged flow fields generated by (a) MCPD fluid simulations of a cell attached to a wall (b) SD simulations of a helix rotating in a bulk fluid computed in the $x y$ plane. These two measured fields differ only by Gaussian fluctuations inherent in the MPCD simulation as shown in (c). [(d)-(f)] Same as panels (a)-(c) except in $x z$ plane. Panel (f) shows that there are systematic differences in the flow fields in this plane, mainly due to the presence of the wall. The contour-enclosed region in the lower quarter of the image represents where the flow field differs by $\left|\bar{v}_{z}^{\mathrm{MPCD}}-\bar{v}_{z}^{\mathrm{SD}}\right|>0.25$. Everywhere else, the fields behave similarly.

remain force free too. Hence, the force and torque nature of our model is more akin to systems in which flagellated cells are in some way stalled, tethered, or otherwise sessile, e.g., by hydrodynamic accumulation at boundaries [39], immobilization on microscope slides [28,29], or as part of their biological function $[17,18]$. However, in these cases, hydrodynamic interactions with the boundaries and cell bodies are a potential source of discrepancy between the results of our model and these experimental or biological systems.

We wish to quantify this difference and demonstrate that our system is nevertheless a sufficiently good model for studying the near-flagellum dynamics of a polymer. To achieve this, we compare the flow fields measured from the SD simulations to multiparticle collision dynamics (MPCD) simulations of a more experimentally realistic geometry with a cell body and neighboring wall, as MPCD provides the appropriate machinery for incorporating boundaries and cell-specific body geometry. Further details of the MPCD procedure can be found in the Appendix.

In Fig. 4, we compared the time-averaged flow fields $\bar{v}^{\mathrm{MPCD}}$ and $\bar{v}^{\mathrm{SD}}$ generated by the MPCD and SD simulations respectively. Both quantities were normalized by dividing by the mean velocity 
inside the volume occupied by the helix. By visual inspection, we observe that the coarse structure of the axial and transverse flows are qualitatively similar in both simulations.

To quantitatively compare the two fields, we analyzed the square differences $\left(\bar{v}_{z, \perp}^{\mathrm{MPCD}}-\bar{v}_{z, \perp}^{\mathrm{SD}}\right)^{2}$ to see how they decay relative to one another. In Fig. 4(c), we can see that $\left(\bar{v}_{\perp}^{\mathrm{MPCD}}-\bar{v}_{\perp}^{\mathrm{SD}}\right)^{2}$ appears to have the structure of uniform noise. A Kolmogorov-Smirnov test on the data for the unsquared difference $\bar{v}_{\perp}^{\mathrm{MPCD}}-\bar{v}_{\perp}^{\mathrm{SD}}$ did not yield evidence for a non-Gaussian distribution, and so we conclude that $\bar{v}_{\perp}^{\mathrm{MPCD}}$ differs from $\bar{v}_{\perp}^{\mathrm{SD}}$ by the Gaussian noise present in the MPCD simulation only.

However, there are more significant differences in the radial flow fields due to the presence of the cell body and the wall in the MPCD simulations. The square difference $\left(\bar{v}_{z}^{\mathrm{MPCD}}-\bar{v}_{z}^{\mathrm{SD}}\right)^{2}$, plotted in Fig. 4(f), reveals a systematic variation across the whole region that is roughly one order of magnitude greater than the noise in Fig. 4(c). The contour-enclosed area connected to the wall (at $z=0$ ) shows the region in which the flow fields differ by $\left|\bar{v}_{z}^{\mathrm{MPCD}}-\bar{v}_{z}^{\mathrm{SD}}\right|>0.25$. Evidently, the cell body and wall have some significant influence on the flow in this region but not in the immediate vicinity of the model flagellum.

The fact that the radial fields are in better agreement than the axial fields can be understood by noting that $\bar{v}_{\perp} \sim 1 / r^{2}$ decays over shorter distances than $\bar{v}_{z} \sim 1 / r$, and hence the wall effects play a much larger role for the axial fields.

Since our main focus in this paper is the near-flagellum dynamics of polymers and helices interacting across length scales similar to and less than their own spatial dimensions, we take these MPCD results as evidence that far-field effects (such as those generated by counter-rotating cell bodies, solid boundaries, and indeed other nearby swimmers or filaments) do not contribute appreciably to the dynamics of polymers sufficiently close to the helical filament. In this region, we expect the chiral, high-shear, and geometry-specific flow of the helix to characterize the behavior of a nearby polymer, and far-field effects due to boundaries or other bodies to be secondary.

\section{POLYMER CAPTURE}

The central result of this paper is that a rotating helix transports a polymer along with the fluid it pumps, but in such a way that a polymer initially on the outside of the helix is drawn inward and "captured" by the helix. This is accompanied by an initial stretching out of the polymer as it migrates toward the helix, moving into a region of greater shear and greater flow as seen in Fig. 1. As it is transported along the interior of the helix, it occasionally wraps around the helical filament and rotates along with it. An instance of such wrapping is observed in Fig. 1. The polymer is deposited at the end of the helix, where a decaying axial current keeps moving it at a diminishing rate, while the lower shear results in the polymer collapsing back into its unstretched ground state. Two movies of a typical capturing process can be found in Ref. [40].

The polymer capture and transport is a stochastic, nonequilibrium transient process; however, by performing a large number of "scattering" simulations and averaging over these, we are able to quantify the typical nature of the interaction as a function of polymer size. We present batches of simulations for degrees of polymerization (number of beads) $N_{p}=1,10,30,50$ - where a polymer of contour length 1 is simply a spherical monomer. In each of these simulations, we use helices with the same parameters: $N_{h}=200$ beads, $\kappa=2 \pi / 15 \sigma, R=4 \sigma$. The helix is centered along the $z$ axis, with its two ends located at $\left(z_{0}, z_{N_{h}-1}\right)=(-57 \sigma, 57 \sigma)$. In each simulation, one polymer is initialized by placing its first monomer randomly on a disk of radius $16 \sigma$, located at $\left(z_{0}-30 \sigma\right)$, then performing a self-avoiding random walk to build the polymer bead by bead. We run each simulation for $\mathcal{T}=2 \times 10^{6} \delta t$, which is sufficient in all cases to advect the polymers beyond the rear of the helix. We measure the relaxation times of the polymers in separate simulations and found them to be $\tau_{p} \approx$ $\{1,10,20\} \times 10^{6} \delta t$ for the $N=\{10,30,50\}$ polymers respectively. Taking the helix rotation speed $\omega=2 \times 10^{-4} / \delta t$ as the characteristic shear rate for the fluid, we therefore estimate the respective Weissenberg numbers characterizing each set of simulations to be $\mathrm{Wi} \sim \omega \tau_{p}=\{200,2000,4000\}$. As Wi $\gg 1$ in each of these cases, the influence of shear flow dominates the polymers' tendency to relax. 

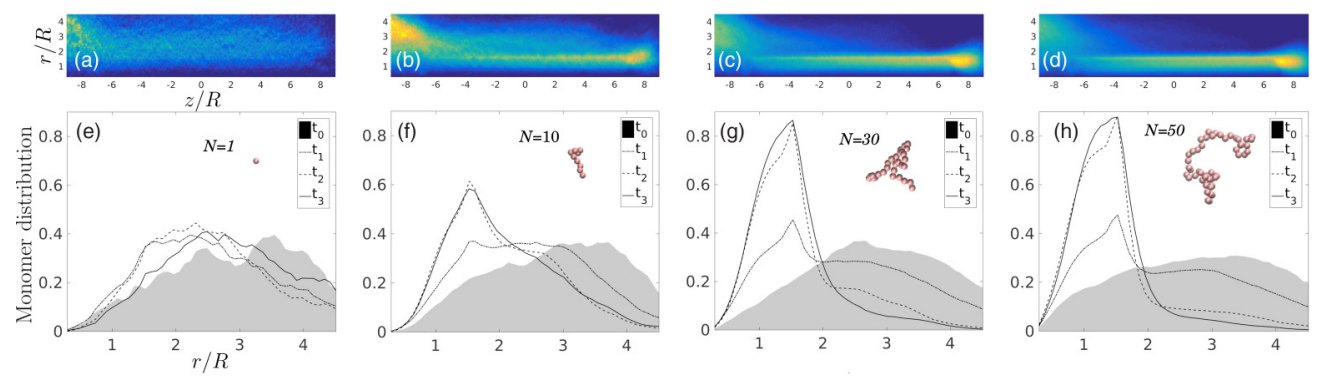

FIG. 5. [(a)-(d)] Polymer distributions averaged over all simulations and over all time show the net behavior of polymers being pumped in the positive- $z$ direction, given an initial distribution of polymers on a disk of radius $r=4 R$, located at $z \approx-10 R$ in cylindrical coordinates $(r, z)$. Each distribution is averaged over 200 simulations for polymers of size (a) $N=1$ (colloidal tracers), (b) $N=10$, (c) $N=30$, and (d) $N=50$. The net behavior is a drift toward the right of each image, due to the helix (not shown) pumping the fluid. [(e)-(h)] The same data as in panels (a)-(c) respectively, but plotted as distributions over $r$ only, with each curve representing contiguous quarter intervals of the simulation time. In each case, the initial quarter is the shaded region, and we observe that for increasing polymer size, a strong tendency for the polymers to migrate inward is observed. For larger polymers, this tendency is stronger and occurs faster.

To illustrate how the ensembles of polymers evolve in time, we plot the average monomer distribution in cylindrical coordinates $(r, z)$ taken over all simulations and at all times. These are plotted for polymers of differing size in Figs. 5(a)-5(d). These images represent time- and ensembleaveraged 2D histograms of the snapshots shown in Fig. 1. At the left edge of each of the images is the initial distribution of polymeric material which in each case is smeared rightward in time. The structure of this distribution gives a graphical indication that larger polymers [such as in Figs. 5(c) and $5(\mathrm{~d})$ ] are much more strongly attracted to the helix than smaller polymers and are highly concentrated in the region $r<2 R$. On the other hand, in Fig. 5(a) this effect is barely observed for $N=1$ monomers, which are simply advected along the streamlines shown in Fig. 2(f) like tracer particles that cannot cross streamlines; polymers, on the other hand, are able to cross streamlines in shear flow [41], and in this case do so strongly in a nontrivial manner.

Particularly for high Weissenberg numbers [42-44], we observe hotspots in the distributions located at the downstream end of the helix. These indicate the accumulation of polymers in this location when they are deposited at the end of the helix and collapse back into their equilibrium conformation. Though they continue moving along the $z$ axis, they do so at a lower rate than when inside the helix. This is due to the fact that the axial flow $\boldsymbol{v}_{z}$ quickly decreases outside of the helix [as can be seen in Fig. 2(f)].

In Figs. 5(e)-5(h), we segment each simulation into four equal and contiguous intervals in time, and separately plot the marginal distributions over $r$ only (i.e., with the $z$ component integrated out) for each time. The shaded region represents the interval $t_{0}=[0, \mathcal{T} / 4]$, which is the first quarter of each simulation and closely approximates the initial distribution. These figures show how the initial distribution evolves with time for polymers of different size. For $N_{p}=30$ and $N_{p}=50$, the tendency to concentrate in and around the helix is markedly stronger than for shorter polymers. This ensemble behavior shows that the actuation of the helix is responsible for a large density fluctuation in the surrounding polymeric material that concentrates - rather than depletes - the polymers in the immediately surrounding region. Animations of the densities as a function of time can be found in Ref. [45].

This implies that the free energy of the polymers must be actively driven away from what we would expect in equilibrium. We obtain an intuitive sense that this is occurring by considering the snapshots in Fig. 1. Initially, the polymer is far away and its configuration is that of a self-avoiding random walk. However, at intermediate times, the polymer is stretched out of equilibrium by the shear flow and is transported radially inward as well as along $z$ until it strongly interacts with the 
helix, wrapping around it and continuing to move along $z$. In the vicinity of the helix, polymers lose their equilibrium conformation, and we observe features in their dynamics similar to those previously reported for polymers in shear flow due to a rotating microwire [42]. To gain further insight into the energetic interplay within our system, we analyze the stochastic fluctuations in work performed by the helix on the polymer.

\section{FLUCTUATING WORK}

Polymers tend to become stretched when immersed in a shear flow, and this agrees with the current SD simulations. However, because the shear flow in this case is generated by external forces acting on the helix, we expect these forces to be dependent on the proximity of a polymer in the surrounding medium. The hydrodynamic origin of this is due to the effect of the polymer on the helix particles: Hydrodynamic interactions displace the helix particles from their steady state positions, resulting in a change in the forces acting on them due to Eq. (7). While these displacements are too small to give rise to a perceptible change in helix shape, they should in general manifest themselves as a change in work that the helix must do to maintain steady rotation.

The work applied to the helix by the external forces, $w[t, \boldsymbol{r}(t)]$, is a fluctuating quantity and a unique function for each realization of a stochastic dynamical process. Work is performed either by the application of a nonconservative force or by a time-varying potential, $\mathcal{H}(\boldsymbol{r}, \lambda(t))$ with an external control parameter $\lambda(t)$. For the latter case, the work applied by a time $t$ is defined by Ref. [46]: $w[t, \boldsymbol{r}(t)]=\int_{0}^{t} d t^{\prime} \dot{\lambda} \partial \mathcal{H} / \partial \lambda$. The nonstochastic forces in our simulation are due to the potentials $H_{\text {repel }}, H_{\text {bond }}$, and $\mathcal{H}_{h}$ in Eqs. (4), (5), and (7). Of these, only the forces acting on the helix due to $\mathcal{H}_{h}$ depend explicitly on time and it is these that are entirely responsible for the work done on the system.

For each simulation, we calculate the incremental work performed by the helix at each time step by

$$
\delta w=\sum_{i} \boldsymbol{f}_{i}^{h} \cdot \delta \boldsymbol{r}_{i}^{0}
$$

where $\boldsymbol{f}_{i}^{h}=-\nabla \mathcal{H}_{h}\left(\boldsymbol{r}_{i}, t\right)$ is time-varying force applied to particle $i$. Note the increment $\delta \boldsymbol{r}_{i}^{0}$ is the displacement of the bead target position, not the displacement of the bead itself. From these increments $\delta w$, we build up an accumulated work trajectory $w(t)=\sum_{t} \delta w$.

We expect there to be two contributions to the work: $w(t)=w_{0}(t)+w_{\text {ex }}(t)$. The dominant contribution $w_{0}(t)$ is the deterministic work done by the rigid helix on the viscous fluid, which is viscously dissipated. The second contribution is $w_{e x}(t)$, which is the stochastic excess work done on the polymer. By conducting simulations without a polymer, we can measure the dominant viscous contribution, $w_{0}(t)$, and use this to calculate the excess contribution in simulations that do contain a polymer: $w_{e x}(t)=w(t)-w_{0}(t)$.

In Fig. 6, we plot three ensembles of trajectories $w_{e x}(t)$ for the work done by a helix on three sizes of polymer: $N=(10,20,40)$. In each simulation, the polymer is initialised by a self-avoiding random walk starting at $\boldsymbol{r}_{0}=\left[0,15 \sigma,\left(z_{0}-15\right) \sigma\right]$, where $z_{0}$ is the $z$ position of the negative-most particle of the helix which pumps fluid in the positive- $z$ direction. We measure $w_{e x}(t)$ for the entirety of each simulation over a time interval of 80 full rotations. For all simulations, this is enough time to allow for the polymer to relax back to its equilibrium conformation after it has exited the positive- $z$ end of the helix.

These sets of trajectories offer another way to look at the stretching effect: a set of stochastic work trajectories $\left\{w_{i}[t, \boldsymbol{r}(t)]\right\}$ drive the polymer to a higher free energy state though in each instance requiring a different amount of work. The color of each curve denotes the radial point of closest approach of the center-of-mass position of the polymer-i.e., the minimum of $r_{c m}(t) / R$ over all $t$-from which we can see that more work was done on polymers which migrated further in. This indicates that more work must be done on maintaining the stretched-out conformation which polymers adopt in the high-shear region of the helix core. 


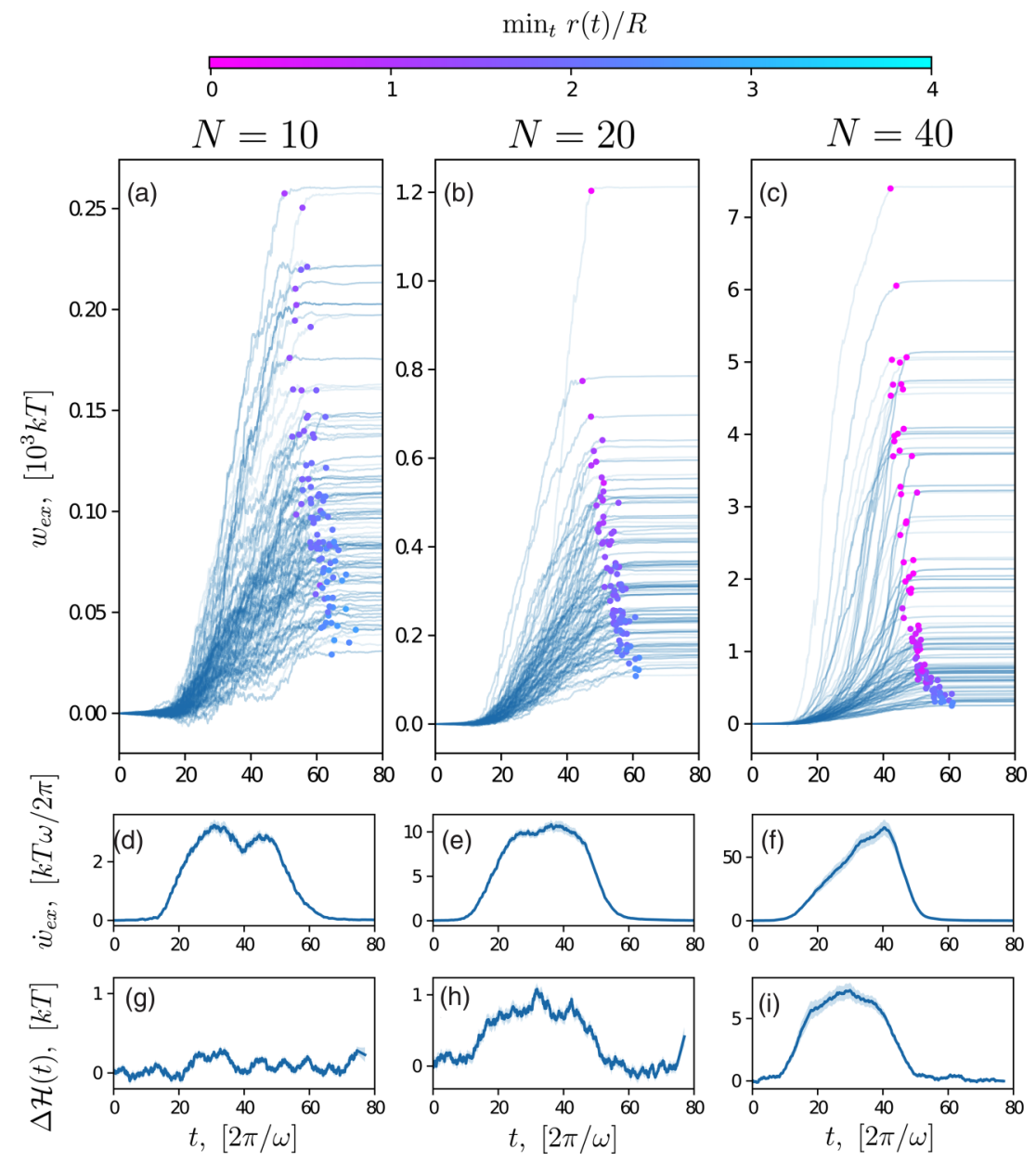

FIG. 6. [(a)-(c)] Stochastic excess work performed by a rotating helix in transporting polymers of size $N=(10,20,40)$ respectively in 200 experiments performed for each case. The markers correspond to the point at which the center of mass of the polymer exits the negative end of the helix. Once this occurs, the polymers collapse and advect with the fluid; no more work is performed on them. The markers are color coded by the point of closest approach to the central long axis of the helix for the polymer in that experiment, $\min _{t}(r)$. More work on average was performed to transport polymers that migrated nearer (magenta) the central axis than on polymers which failed to become captured (cyan). [(d)-(f)] Mean excess power per revolution, averaged over 200 simulations. For each time step, $d w_{e x}$ was calculated and smoothed with a window size of $\sim 1 / 4$ revolution. The shaded region corresponds to the standard error on the mean. [(g)-(i)] Mean increase in polymer energy calculated using the potentials $H_{\text {repel }}$ and $H_{\text {bond }}$ with the same smoothing and averaging procedure employed in panels (d)-(f).

The trajectories are nonmonotonic, and some trajectories temporarily deviate into the negative work region, which is a hallmark of the thermodynamics of stochastic systems. We further notice that once the polymer has exited the helix, $w_{e x}(t)$ flattens dramatically. This corresponds to the observation that when the polymer is deposited at the rear end of the helix, it quickly collapses to its equilibrium configuration and highlights that the rotation of the helix principally impacts polymers in its immediate vicinity. Furthermore, the fact that $w_{e x}(t)$ does not overshoot its final value tells us that the helix does not elastically regain any of the work it has supplied to the polymer when the polymer relaxes. Indeed, the scale of $w_{\mathrm{ex}}$ is much larger than the stored energy in the polymer, suggesting that this energy is being dissipated into the environment. This is in contrast to the observed enhancement 
of swimming due to the energetics of noiseless elastic surroundings $[47,48]$. In such systems, elastic networks or tubes which a swimmer swims through store elastic energy and transfer this energy back to during relaxation. However, in our system, the heat bath to which the polymer is attached robs the helix of any such energy storage mechanism.

To gain further insight, we measured the change in energy stored in the polymers, $\Delta \mathcal{H}(t)=$ $\Delta H_{\text {bond }}(t)+\Delta H_{\text {repel }}(t)$ (where $\Delta$ corresponds to a difference with respect to the quantities evaluated at $t=0$ ). After smoothing these traces using a Savitzky-Golay filter with a window size of $\sim 1 / 4$ helix revolutions, and then averaging over all simulations, we obtain an indication of how the energy stored in the polymer increases on average. We also applied the same smoothing and averaging procedure to the work increments $d w_{e x}(t)$ to compare how the mean energy stored in the polymers was related to the work rate of the helix. These results are plotted in Figs. 6(d)-6(i). We see no measurable increase in stored energy for the $N=10$ polymer, but statistically significant increases for the $N=20$ and $N=40$ polymers. These increases in energy are due to the elongation the larger polymers suffer when they are captured by the helix - a phenomenon which we have shown varies with polymer size. While the energy stored in the $N=10$ polymer does not significantly increase, the helix still performs a significant amount of work in transporting it. For the $N=20$ and $N=50$ polymers, we can see that the helix performs roughly $10 \mathrm{k}_{B} T$ of work per revolution for every $1 \mathrm{k}_{B} T$ energy maintained in the polymer. Because for the $N=10$ polymer the power is on the order of $1 \mathrm{k}_{B} T$ per revolution, this predicts the mean stored energy to be much less than the its fluctuations and hence negligible.

In general, the work excess term is typically smaller than the viscous term by $\sim 4$ orders of magnitude. However, this is for a solitary polymer in the vicinity of the helix. In a suspension of polymers, we hypothesize that the excess work takes the form of a sum over the work performed on each polymer and so will be proportional to the local density of the solution at least in the dilute limit where polymer-polymer interactions can be ignored. As we have shown separately, the effect of the helix is to increase the local density of polymeric material so we expect this, combined with the work done on stretching the polymers, to give rise to strongly nonlinear viscoelastic effects. This offers some contrast to the hypothesis that bacterial flagella on their own deplete their local environment of biopolymeric material and hence experience only the background Newtonian solvent [12].

\section{CONCLUSIONS}

Microbes live in complex fluidic environments, often of their own making. Microbial extracellular polymeric substances are continually secreted for a wide variety of purposes [49,50], including anchoring to surfaces by long mucous stalks [18,19], bioaccumulation of contaminants [51], and to serve as the polymeric matrix within biofilms [52], veils [53], and other collective structures [54]. Motile swimmers must move through these complex media, while sessile microorganisms drive the transport of large high-conformational-entropy biopolymers. Previous work has focused on feeding currents [13] entraining nutrients modelled as tracer particles (which can be well described by hydrodynamic multipole expansion methods) $[55,56]$ or on the continuum limit of a viscoelastic fluid medium through which microbes must swim [1,7,57-59] or pump [60,61] fluid. Both approaches average over the many internal degrees of freedom of long, flexible biopolymers.

We have shown how Stokesian dynamics simulations of a rotating helical filament can accurately model the near-field fluid flow of a flagellated cell tethered to a wall and studied the effects of this flow field on nearby coarse-grained polymers of various size. We have shown that long polymers are strongly attracted to the model flagellum and undergo a nonequilibrium stretching process as they are pulled toward it and pumped along it. This implies that a dilute suspension of polymers tend to become locally concentrated in and around the flagellum rather than depleted.

Our results show that it is possible in simulations to measure the work applied to the polymer by the helix and that this is on average positive. Moreover, all of the work supplied to the polymer is dissipated, meaning that there is no elastic reclamation by the helix of the polymer's free energy when it collapses upon exit of the helix such as that observed in noiseless systems [47,48]. Hence, our results provide some fundamental phenomenological insights into activity in microscopic, viscoelastic 
systems. In future work, we hope to tackle the question of a swimming helix (i.e., one in which $v_{z}$ is set to a speed that ensures the force-free condition: $f_{z}=0$ ), as well as testing the hypothesis that multiple polymers increase the excess work done by the helix by a proportionate amount.

\section{ACKNOWLEDGMENTS}

This work was supported through funding from the ERC Advanced Grant No. 291234 MiCE and we acknowledge EMBO funding to T.N.S. (ALTF181-2013). A.Z. acknowledges funding by Marie Skłodowska Curie Intra-European Fellowship (G.A. No. 653284) within Horizon 2020. We thank A. Petroff and A. Libchaber for introducing us to veil-forming microbes.

\section{APPENDIX: MULTIPARTICLE COLLISION DYNAMICS SIMULATION}

To verify that our SD helix model well approximates the near-field flow of a sessile flagellated microbe in the presence of environmental boundaries in a manner that does not depend significantly on cell-body-induced or wall-induced hydrodynamic interactions, we construct a more biologically accurate but computationally costly model of a wall-tethered bacterium and simulate it using multiparticle collision dynamics (MPCD).

MPCD is a particle-based method to solve the Navier-Stokes equations on a coarse-grained level where particle dynamics and interactions are solved in alternating streaming and collision steps $[62,63]$. This method has been used successfully to model the hydrodynamics of microswimmers near surfaces [64-71] and wall-tethered flagella [72,73]. The fluid is modeled by pointlike effective particles with mass $m$ at positions $\boldsymbol{r}_{i}$ with velocities $\boldsymbol{v}_{i}$.

In the streaming step, the fluid particles move ballistically for a time step $\delta t$, and their positions are updated according to

$$
\boldsymbol{r}_{i}(t+\delta t)=\boldsymbol{r}_{i}(t)+\boldsymbol{v}_{i}(t) \delta t .
$$

In the collision step, particles are sorted into cubic cells of side length $h$, and all particles in a cell stochastically exchange momentum according to

$$
\boldsymbol{v}_{i}(t+\delta t)=\boldsymbol{u}_{\xi}+\boldsymbol{v}_{r}+\boldsymbol{v}_{P}+\boldsymbol{v}_{L}
$$

where $\boldsymbol{u}_{\xi}$ is the mean velocity in the cell, $\boldsymbol{v}_{r}$ is a random velocity drawn from a Maxwell-Boltzmann distribution at temperature $T$, and $\boldsymbol{v}_{P}$ and $\boldsymbol{v}_{L}$ are correction factors to conserve momentum and angular momentum in the cell [74]. All physical quantities are measured in units of cell length $h$, fluid mass $m$, and thermal energy $k_{B} T$. We use a time step $\delta t=0.02 \sqrt{m h^{2} / k_{B} T}$ and a mean number of fluid particles per cell $\gamma=10$, resulting in high Schmidt and Mach numbers to reproduce near-incompressible viscous Newtonian flows at low Reynolds number [75].

Figure 4(d) includes a representation of the bacterium model we use. The cell body itself is modeled as a rigid superellipsoid [76] defined by the surface $\left[\left(x / h_{x}\right)^{2 / \epsilon_{2}}+\left(y / h_{y}\right)^{2 / \epsilon_{2}}\right]^{\epsilon_{2} / \epsilon_{1}}+$ $\left(z / h_{z}\right)^{2 / \epsilon_{1}}=1$, where we use $h_{x}=h_{y}=2 h, h_{z}=4 h, \epsilon_{1}=0.5$, and $\epsilon_{2}=1$. It is oriented perpendicular to a wall (located at $z=-50 h)$ and fixed at $\left(x_{0}, y_{0}, z_{0}\right)=(0,0,-46 h)$. We add a second wall far away from the bacterium at $z=50 h$, and use periodic boundary conditions in the $x$ and $y$ directions with $x, y, \in(-50 h, 50 h)$. The flagellum is modeled as a rigid helical polymer consisting of 57 pointlike beads of mass $10 \mathrm{~m}$, which are separated by $1 h$. The helix is given by the curve

$$
\boldsymbol{r}(z)=\left(R\left[1-e^{-\left(\kappa z / l_{s}\right)^{2}}\right] \cos (\kappa z), R\left[1-e^{-\left(\kappa z / l_{s}\right)^{2}}\right] \sin (\kappa z), z\right),
$$

where we use $R=2 h$ as the helix radius, pitch $\kappa=1 / h$, and $\kappa z$ is the phase of the helix measured from where it meets the body. A nonzero Higdon parameter [77] $l_{s}=3$ ensures that the helix is attached at the center of the cell body surface.

To model no-slip boundary conditions at the walls and the cell surface, a bounce-back rule for the fluid particles is used [63]. The transfer of momentum between the flagellum and the fluid is achieved 


\section{BALIN, ZÖTTL, YEOMANS, AND SHENDRUK}

by including the flagellum beads into the collision step [78]. While the cell body is kept fixed during the simulation, the helix is rotated with a constant angular velocity $\omega=\left(5.6 \times 10^{-4}\right) / \delta t$, and the surrounding flow field is measured for a time $t=10^{5} \delta t$ and averaged over 70 independent runs.

[1] B. Liu, T. R. Powers, and K. S. Breuer, Force-free swimming of a model helical flagellum in viscoelastic fluids, Proc. Nat. Acad. Sci. USA 108, 19516 (2011).

[2] D. A. Gagnon, X. Shen, and P. E. Arratia, Undulatory swimming in fluids with polymer networks, EPL 104, 14004 (2013).

[3] S. E. Spagnolie, B. Liu, and T. R. Powers, Locomotion of Helical Bodies in Viscoelastic Fluids: Enhanced Swimming at Large Helical Amplitudes, Phys. Rev. Lett. 111, 068101 (2013).

[4] B. Thomases and R. D. Guy, Mechanisms of Elastic Enhancement and Hindrance for Finite-Length Undulatory Swimmers in Viscoelastic Fluids, Phys. Rev. Lett. 113, 098102 (2014).

[5] E. E. Riley and E. Lauga, Enhanced active swimming in viscoelastic fluids, EPL 108, 34003 (2014).

[6] A. E. Patteson, A. Gopinath, M. Goulian, and P. E. Arratia, Running and tumbling with E. coli in polymeric solutions, Sci. Rep. 5, 15761 (2015).

[7] E. Lauga, Propulsion in a viscoelastic fluid, Phys. Fluids 19, 083104 (2007).

[8] H. C. Fu, T. R. Powers, and C. W. Wolgemuth, Theory of Swimming Filaments in Viscoelastic Media, Phys. Rev. Lett. 99, 258101 (2007).

[9] X. N. Shen and P. E. Arratia, Undulatory Swimming in Viscoelastic Fluids, Phys. Rev. Lett. 106, 208101 (2011).

[10] L. Zhu, E. Lauga, and L. Brandt, Self-propulsion in viscoelastic fluids: Pushers vs. pullers, Phys. Fluids 24, 051902 (2012).

[11] B. Qin, A. Gopinath, J. Yang, J. P. Gollub, and P. E. Arratia, Flagellar kinematics and swimming of algal cells in viscoelastic fluids, Sci. Rep. 5, 9190 (2015).

[12] V. A. Martinez, J. Schwarz-Linek, M. Reufer, L. G. Wilson, A. N. Morozov, and W. C. K. Poon, Flagellated bacterial motility in polymer solutions, Proc. Nat. Acad. Sci. USA 111, 17771 (2014).

[13] J. J. L. Higdon, The generation of feeding currents by flagellar motions, J. Fluid Mech. 94, 305 (1979).

[14] K. C. Marshall, Planktonic versus sessile life of prokaryotes, in The Prokaryotes: Prokaryotic Communities and Ecophysiology, edited by E. Rosenberg, E. F. DeLong, S. Lory, E. Stackebrandt, and F. Thompson (Springer, Berlin, 2013), pp. 191-201.

[15] E. Lauga and T. R. Powers, The hydrodynamics of swimming microorganisms, Rep. Prog. Phys. 72, 096601 (2009).

[16] J. Elgeti, R. G. Winkler, and G. Gompper, Physics of microswimmers-single particle motion and collective behavior: A review, Rep. Prog. Phys. 78, 056601 (2015).

[17] R. E. Pepper, M. Roper, S. Ryu, P. Matsudaira, and H. A. Stone, Nearby boundaries create eddies near microscopic filter feeders, J. R. Soc. Interf. 7, 851 (2009).

[18] A. P. Petroff, A. L. Pasulka, N. Soplop, X.-L. Wu, and A. Libchaber, Biophysical basis for convergent evolution of two veil-forming microbes, R. Soc. Open Sci. 2, 150437 (2015).

[19] A. P. Petroff and A. Libchaber, Hydrodynamics and collective behavior of the tethered bacterium Thiovulum majus, Proc. Nat. Acad. Sci. USA 111, E537 (2014).

[20] J. S. Guasto, R. Rusconi, and R. Stocker, Fluid mechanics of planktonic microorganisms, Annu. Rev. Fluid Mech. 44, 373 (2012).

[21] D. L. Ermak and J. A. McCammon, Brownian dynamics with hydrodynamic interactions, J. Chem. Phys. 69, 1352 (1978).

[22] J. Rotne and S. Prager, Variational treatment of hydrodynamic interactions in polymers, J. Chem. Phys. 50, 4831 (1969).

[23] H. Yamakawa, Transport properties of polymer chains in dilute solution: Hydrodynamic interaction J. Chem. Phys. 53, 436 (1970).

[24] G. W. Slater, C. Holm, M. V. Chubynsky, H. W. de Haan, A. Dubé, K. Grass, O. A. Hickey, C. Kingsburry, D. Sean, T. N. Shendruk, and L. Zhan, Modeling the separation of macromolecules: A review of current computer simulation methods, Electrophoresis 30, 792 (2009). 


\section{BIOPOLYMER DYNAMICS DRIVEN BY HELICAL FLAGELLA}

[25] G. S. Grest and K. Kremer, Molecular dynamics simulation for polymers in the presence of a heat bath, Phys. Rev. A 33, 3628 (1986).

[26] The pitch as usually defined as the length of a single complete turn, which in our notation is $2 \pi / \kappa$.

[27] J. Hu, M. Yang, G. Gompper, and R. G. Winkler, Modelling the mechanics and hydrodynamics of swimming E. coli, Soft Matter 11, 7867 (2015).

[28] J. Yuan and H. C. Berg, Following the behavior of the flagellar rotary motor near zero load, Exp. Mech. 50, 1263 (2010).

[29] J. Xing, F. Bai, R. Berry, and G. Oster, Torque-speed relationship of the bacterial flagellar motor, Proc. Nat. Acad. Sci. USA 103, 1260 (2006).

[30] M. J. Kim, J. C. Bird, A. J. Van Parys, K. S. Breuer, and T. R. Powers, A macroscopic scale model of bacterial flagellar bundling, Proc. Nat. Acad. Sci. USA 100, 15481 (2003).

[31] M. J. Kim, M. J. Kim, J. C. Bird, J. Park, T. R. Powers, and K. S. Breuer, Particle image velocimetry experiments on a macro-scale model for bacterial flagellar bundling, Exp. Fluids 37, 782 (2004).

[32] T. S. Yu, E. Lauga, and A. E. Hosoi, Experimental investigations of elastic tail propulsion at low Reynolds number, Phys. Fluids 18, 091701 (2006).

[33] N. Coq, O. du Roure, M. Fermigier, and D. Bartolo, Helical beating of an actuated elastic filament, J. Phys.: Condens. Matter 21, 204109 (2009).

[34] S. Zhong, K. W. Moored, V. Pinedo, J. Garcia-Gonzalez, and A. J. Smits, The flow field and axial thrust generated by a rotating rigid helix at low Reynolds numbers, Exp. Therm. Fluid Sci. 46, 1 (2013).

[35] J. Lighthill, Flagellar hydrodynamics, SIAM Rev. 18, 161 (1976).

[36] B. Rodenborn, C.-H. Chen, H. L. Swinney, B. Liu, and H. P. Zhang, Propulsion of microorganisms by a helical flagellum, Proc. Nat. Acad. Sci. USA 110, E338 (2013).

[37] W. Gao, X. Feng, A. Pei, C. R. Kane, R. Tam, C. Hennessy, and J. Wang, Bioinspired helical microswimmers based on vascular plants, Nano Lett. 14, 305 (2014).

[38] K. E. Peyer, L. Zhang, and B. J. Nelson, Bio-inspired magnetic swimming microrobots for biomedical applications, Nanoscale 5, 1259 (2013).

[39] S. E. Spagnolie and E. Lauga, Hydrodynamics of self-propulsion near a boundary: Predictions and accuracy of far-field approximations, J. Fluid Mech. 700, 105 (2012).

[40] See Supplemental Material at http://link.aps.org/supplemental/10.1103/PhysRevFluids.2.113102 for two videos of a typical capture process.

[41] M. D. Graham, Fluid dynamics of dissolved polymer molecules in confined geometries, Annu. Rev. Fluid Mech. 43, 273 (2011).

[42] T. N. Shendruk, D. Sean, D. J. Berard, J. Wolf, J. Dragoman, S. Battat, G. W. Slater, and S. R. Leslie, Rotation-induced macromolecular spooling of DNA, Phys. Rev. X 7, 031005 (2017).

[43] A. R. Klotz, V. Narsimhan, B. W. Soh, and P. S. Doyle, Dynamics of DNA knots during chain relaxation, Macromolecules 50, 4074 (2017).

[44] R. G. Winkler, Conformational and rheological properties of semiflexible polymers in shear flow, J. Chem. Phys. 133, 164905 (2010).

[45] See Supplemental Material at http://link.aps.org/supplemental/10.1103/PhysRevFluids.2.113102 for animations of the density functions as a function of time.

[46] U. Seifert, Stochastic thermodynamics, fluctuation theorems and molecular machines, Rep. Prog. Phys. 75, 126001 (2012).

[47] R. Ledesma-Aguilar and J. M. Yeomans, Enhanced Motility of a Microswimmer in Rigid and Elastic Confinement, Phys. Rev. Lett. 111, 138101 (2013).

[48] J. K. Wrobel, S. Lynch, A. Barrett, L. Fauci, and R. Cortez, Enhanced flagellar swimming through a compliant viscoelastic network in Stokes flow, J. Fluid Mech. 792, 775 (2016).

[49] A. W. Decho, Microbial exopolymer secretions in ocean environments: Their role(s) in food webs and marine processes, Oceanogr. Mar. Biol. Annu. Rev. 28, 73 (1990).

[50] G. M. Wolfaardt, J. R. Lawrence, and D. R. Korber, Function of EPS, in Microbial Extracellular Polymeric Substances (Springer, Berlin, 1999), pp. 171-200.

[51] G. M. Wolfaardt, J. R. Lawrence, J. V. Headley, R. D. Robarts, and D. E. Caldwell, Microbial exopolymers provide a mechanism for bioaccumulation of contaminants, Microbial Ecol. 27, 279 (1994). 
[52] H.-C. Flemming, T. R. Neu, and D. J. Wozniak, The EPS matrix: The "house of biofilm cells," J. Bacteriol. 189, 7945 (2007).

[53] C. O. Wirsen and H. W. Jannasch, Physiological and morphological observations on Thiovulum sp, J. Bacteriol. 136, 765 (1978).

[54] C. Schaudinn, P. Stoodley, A. Kainović, T. O'Keeffe, B. Costerton, D. Robinson, M. Baum, G. Ehrlich, and P. Webster, Bacterial biofilms, other structures seen as mainstream concepts, Microbe 2, 231 (2007).

[55] A. J. T. M. Mathijssen, D. O. Pushkin, and J. M. Yeomans, Tracer trajectories and displacement due to a micro-swimmer near a surface, J. Fluid Mech. 773, 498 (2015).

[56] A. J. T. M. Mathijssen, A. Doostmohammadi, J. M. Yeomans, and T. N. Shendruk, Hydrodynamics of micro-swimmers in films, J. Fluid Mech. 806, 35 (2016).

[57] B. Liu, M. Gulino, M. Morse, J. X. Tang, T. R. Powers, and K. S. Breuer, Helical motion of the cell body enhances Caulobacter crescentus motility, Proc. Nat. Acad. Sci. USA 111, 11252 (2014).

[58] E. Lauga, Locomotion in complex fluids: Integral theorems, Phys. Fluids 26, 081902 (2014).

[59] A. E. Patteson, A. Gopinath, and P. E. Arratia, Active colloids in complex fluids, Curr. Opin. Colloid Interface Sci. 21, 86 (2016).

[60] M. A. Sleigh, J. R. Blake, and N. Liron, The propulsion of mucus by cilia, Am. Rev. Respir. Dis. 137, 726 (1988).

[61] J. R. Vélez-Cordero and E. Lauga, Waving transport and propulsion in a generalized Newtonian fluid, J. Non-Newtonian Fluid Mech. 199, 37 (2013).

[62] R. Kapral, Multiparticle collision dynamics: Simulation of complex systems on mesoscales, Adv. Chem. Phys. 140, 89 (2008).

[63] G. Gompper, T. Ihle, D. M. Kroll, and R. G. Winkler, Multi-particle collision dynamics: A particle-based mesoscale simulation approach to the hydrodynamics of complex fluids, Adv. Polym. Sci. 221, 1 (2009).

[64] J. Elgeti and G. Gompper, Self-propelled rods near surfaces, EPL 85, 38002 (2009).

[65] J. Elgeti, U. B. Kaupp, and G. Gompper, Hydrodynamics of sperm cells near surfaces, Biophys. J. 99, 1018 (2010).

[66] A. Zöttl and H. Stark, Nonlinear Dynamics of a Microswimmer in Poiseuille Flow, Phys. Rev. Lett. 108, 218104 (2012).

[67] A. Zöttl and H. Stark, Hydrodynamics Determines Collective Motion and Phase Behavior of Active Colloids in Quasi-Two-Dimensional Confinement, Phys. Rev. Lett. 112, 118101 (2014).

[68] K. Schaar, A. Zöttl, and H. Stark, Detention Times of Microswimmers Close to Surfaces: Influence of Hydrodynamic Interactions and Noise, Phys. Rev. Lett. 115, 038101 (2015).

[69] J. Hu, A. Wysocki, R. G. Winkler, and G. Gompper, Physical sensing of surface properties by microswimmers-directing bacterial motion via wall slip, Sci. Rep. 5, 9586 (2015).

[70] M. Theers, E. Westphal, G. Gompper, and R. G. Winkler, Modeling a spheroidal microswimmer and cooperative swimming in a narrow slit, Soft Matter 12, 7372 (2016).

[71] T. Eisenstecken, J. Hu, and R. G. Winkler, Bacterial swarmer cells in confinement: A mesoscale hydrodynamic simulation study, Soft Matter 12, 8316 (2016).

[72] S. Y. Reigh, R. G. Winkler, and G. Gompper, Synchronization and bundling of anchored bacterial flagella, Soft Matter 8, 4363 (2012).

[73] S. Y. Reigh, R. G. Winkler, and G. Gompper, Synchronization, slippage, and unbundling of driven helical flagella, PLoS ONE 8, e70868 (2013).

[74] H. Noguchi, N. Kikuchi, and G. Gompper, Particle-based mesoscale hydrodynamic techniques, EPL 78, 10005 (2007).

[75] J. T. Padding and A. A. Louis, Hydrodynamic interactions and Brownian forces in colloidal suspensions: Coarse-graining over time and length scales, Phys. Rev. E 74, 031402 (2006).

[76] A. H. Barr, Superquadrics and angle-preserving transformations, IEEE Comput. Graph. Appl. 1, 11 (1981).

[77] J. J. L. Higdon, A hydrodynamic analysis of flagellar propulsion, J. Fluid Mech. 90, 685 (1979).

[78] A. Malevanets and J. M. Yeomans, Dynamics of short polymer chains in solution, EPL 52, 231 (2000). 